Alors que la récolte de données et les vérifications de toutes sortes sont à la mode, ô combien! I'Institut suisse pour la formation médicale (ISFM) s'attache à simplifier les procédures de contrôle le plus possible, ce dont chacun pourra être reconnaissant!
En effet, I'informatique et l'Internet offrent des possibilités dont chacun va pouvoir profiter pour alléger un peu sa paperasse, comme Werner Bauer, président de I'ISFM, nous I'explique ci-dessous. Merci à lui de cet éditorial riche en informations importantes!

Dr Jacques de Haller, Président de la FMH

\title{
La formation continue des médecins entre calcul d'apothicaire et responsabilité individuelle
}

Nombre d'entre nous ont des sentiments partagés sur la formation continue réglementaire et le système de crédits annuels. Mais bien évidemment, nous sommes tous conscients $\mathrm{du}$ devoir qui nous incombe de maintenir notre niveau de connaissances. La plupart d'entre nous sont aussi curieux qu'intéressés de découvrir et d'assimiler les nouvelles avancées qu'ils pourront intégrer dans leur pratique quotidienne et de savoir quelles évidences restent de mise. Mais nous savons aussi que les lois et les règlements de même que les programmes de formation continue sont là pour garantir le respect des exigences, aussi par ceux qui ne s'y tiendraient pas de leur propre chef. Cela vaut particulièrement pour la promotion de la qualité, et donc aussi pour la formation continue des médecins. Depuis 2007, celle-ci figure formellement parmi les devoirs professionnels inscrits dans la loi sur les professions médicales. Or, dans la logique des autorités de surveillance, devoir signifie contrôle! Il ne nous reste donc qu'à nous en accommoder, même si parfois, ce comptage de crédits nous horripile. Je reste néanmoins convaincu que la majorité des médecins accomplissent les heures exigées sans cadre arithmétique. Les éventuelles sanctions relèvent elles des autorités cantonales des affaires sanitaires.

\section{La plate-forme de formation continue en ligne facilite les démarches pour les médecins.}

Comment, en collaboration avec les sociétés de discipline médicale, l'Institut suisse pour la formation médicale postgraduée et continue (ISFM) peut-il contribuer à ce que ces calculs d'apothicaire nous importunent le moins possible et que la responsabilité individuelle demeure la clé de voûte de la formation continue?

L'article «Formation continue: le début d'une nouvelle ère» en page 1981 de ce numéro explique comment mettre en œuvre les programmes de formation continue sans tracas administratifs.

En sa qualité de centre de prestations pour les sociétés de discipline médicale, l'ISFM a donc créé une plate-forme de formation continue en ligne (www.fmh.ch $\rightarrow$ ISFM $\rightarrow$ Formation continue $\rightarrow$ Plate-forme de formation continue) permet- tant aux médecins de saisir facilement les sessions suivies, de les envoyer à leur société de discipline et d'imprimer dans la foulée leur diplôme de formation continue. La Société suisse de médecine interne l'a inaugurée avec succès et nous espérons que d'autres sociétés lui emboîteront le pas en 2011. Elles pourront ainsi réduire considérablement leurs charges administratives.

Il sera également plus aisé d'acquérir les 50 crédits annuels exigés ( 150 crédits pour les 3 ans de formation continue réglementaire). Les sociétés médicales définissent la for-

\section{Les sociétés de discipline médicale pourront ainsi réduire leurs charges administratives.}

mation essentielle spécifique exigeant l'obtention de 25 crédits par année. Les 25 autres crédits peuvent être acquis dans le cadre de la formation continue élargie reconnue par d'autres sociétés de discipline, les sociétés cantonales de médecine ou la FMH. A cela s'ajoutent 30 heures d'étude personnelle non soumises à contrôle. Les médecins qui détiennent plus d'un titre accomplissent leur formation continue dans la discipline qui correspond le mieux à leur activité. Les formations approfondies n'ont pas de programme de formation continue propre et la plupart des attestations de formation complémentaire sont soumises à des procédures de recertification réglées en dehors des programmes de formation continue de chaque titre de spécialiste.

En concentrant la Réglementation pour la formation continue sur l'essentiel et en créant la plate-forme de formation continue, l'ISFM propose aux médecins et aux sociétés médicales un outil utile et judicieux pour une formation tout au long de la vie, au sens où Osler l'entendait: «If the license to practise meant the completion of his education how sad it would be for the practitioner, how distressing for his patients! More clearly than any other the physician should illustrate the truth of Plato's saying that education is a life-long process.» (William Osler, Lancet, 1900).

Dr Werner Bauer, président de l'Institut suisse pour la formation médicale (ISFM) 